\title{
A nuclear localization signal is required for the nuclear translocation of Fign and its microtubule-severing function
}

\author{
JIONG LI $^{1 *}$, FENGMING WU ${ }^{1 *}$, LONGFEI CHENG ${ }^{1,2}$, JIAQI ZHANG $^{1,3}$, CAIHUI CHA $^{1,4}$, \\ LI CHEN $^{1}$, TAOSHAN FENG ${ }^{1}$, JIFENG ZHANG ${ }^{1}$ and GUOQING GUO ${ }^{1}$ \\ ${ }^{1}$ Department of Anatomy, Neuroscience Laboratory for Cognitive and Developmental Disorders, \\ Medical College of Jinan University, Guangzhou, Guangdong 510632; ${ }^{2}$ Department of Neurosurgery, \\ The First Affiliated Hospital of Jinan University, Guangzhou, Guangdong 510630; ${ }^{3}$ Department of Neurology, \\ The Affiliated Hospital of Qingdao University, Qingdao, Shandong 266003; ${ }^{4}$ Department of Pediatrics, \\ Guangzhou Women and Children's Medical Center, Guangzhou, Guangdong 510120, P.R. China
}

Received April 6, 2019; Accepted March 4, 2020

DOI: $10.3892 / \mathrm{mmr} .2020 .11040$

\begin{abstract}
It is commonly known that the specific function of a given ATPase associated with diverse cellular activities protein (i.e., a member of the AAA superfamily of proteins) depends primarily on its subcellular location. The microtubule-severing protein fidgetin (Fign) possesses a nuclear localization signal (NLS) that facilitates its translocation to the nucleus, where its assembly is finalized; here, Fign contributes to the regulation of microtubule configuration by cutting and trimming microtubule polymers. In the present study, Fign was found to be a nuclear protein, whose N-terminal sequence (SSLKRKAFYM; residues 314-323) acts as an NLS. Following substitution (KR to NN; 317-318) or deletion (NT; 314-323) mutations within the NLS, Fign, which is predominantly expressed in the nucleus, was found to reside in the cytoplasm of transfected cells. Furthermore, Fign was found to have an essential role in microtubule severing by preferentially targeting highly-tyrosinated microtubules (tyr-MTs). Mutation of the Fign NLS did not affect its microtubule-severing function or the cleavage of tyr-MTs, but did affect the cellular distribution of the Fign protein itself. Taken altogether, an NLS for Fign was identified, and it was demonstrated that the basic amino acids K317 and R318 are necessary for regulating its entry into the nucleus, whereas an increase in Fign in the
\end{abstract}

Correspondence to: Dr Jifeng Zhang or Professor Guoqing Guo, Department of Anatomy, Neuroscience Laboratory for Cognitive and Developmental Disorders, Medical College of Jinan University, 601 Huangpu West Road, Tianhe, Guangzhou, Guangdong 510632, P.R. China

E-mail: tzjf_jennifer@jnu.edu.cn

E-mail: tgqguo@jnu.edu.cn

*Contributed equally

Key words: fidgetin, nuclear localization signal, microtubule severing, tyrosinated modification, microtubule cytosol due to mutations of the NLS did not affect its cleavage function.

\section{Introduction}

The cell cytoskeleton provides shape and structural support $(1,2)$ and consists of microfilaments, intermediate filaments and microtubules. Microtubules and actin filaments cooperate to facilitate the assembly, organization and remodeling of the cytoskeleton, which is critical for different stages of cellular development $(3,4)$. Microtubules perform a variety of functions, including tracking intracellular trafficking, localizing organelles, mediating intracellular signals and maintaining cellular structure (5). Microtubule defects cause various abnormalities and disorders of microtubule-mediated processes; moreover, studies have illustrated that several developmental defects are associated with mutations in microtubule-related genes, such as those of the microtubule-associated proteins, motor-associated regulators, microtubule-severing proteins and microtubule-based motor proteins (6-8). However, a number of the underlying molecular mechanisms regarding the severing of microtubule-based structures have yet to be elucidated.

Fidgetin (Fign), a member of the ATPase associated with diverse cellular activities (AAA) protein family, belongs to the same subfamily as microtubule-severing proteins, such as spastin and katanin (9-14). AAA proteins are involved in a diverse range of cellular activities, including membrane fusion, peroxisome biogenesis, proteasome function, vesicle-mediated transport and microtubule dynamics (15-17). Fign mutations frequently result in developmental abnormalities of the brain, eyes, inner ear and specific bones (12). It is predominantly localized in the nucleus due to the presence of a bipartite nuclear localization signal (NLS) in its central region (11). As reported, the function of Fign is closely associated with its subcellular localization (18); however, the mechanism of nuclear import during these cellular events is poorly understood. Although the majority of microtubule-severing proteins preferentially act on the stable domain of the microtubule, Fign acts at 
the labile domains (19). However, whether an alteration in its cellular distribution affects its microtubule-severing function remains to be elucidated.

Based on the phenotype of Fign-mutant mice, Fign was revealed to be involved in the regulation of signaling pathways, particularly those involved in developmental processes (11). Since the specific function of an AAA protein depends on its interacting partners and precise subcellular location (9), it is important to assess the distribution of Fign and the mechanism of its regulation between the cytoplasm and the nucleus.

In the present study, a Fign mutant was used to confirm its nuclear translocation and microtubule-severing function. An NLS motif was also identified to mediate the nuclear import of the protein. Furthermore, the results indicated that Fign did not sever microtubules into visibly shorter fragments; rather, that Fign and its mutants decreased the number of tyrosinated microtubules (tyr-MTs) at the cell periphery. Collectively, the findings of the present study indicate that Fign is a nuclear protein, and that the basic amino acids K317 and R318 within its NLS are key to its nuclear localization and microtubule-severing function.

\section{Materials and methods}

Reagents. The pEGFP-N1 (cat. no. 6085-1; Addgene, Inc.) plasmid was maintained within our laboratory. Normal Donkey serum (cat. no. D9663) was purchased from Sigma-Aldrich; Merck KGaA. Opti-MEM, DMEM high glucose medium, trypsin and FBS were purchased from Gibco; Thermo Fisher Scientific, Inc., and Lipofectamine ${ }^{\circledR} 2000$ and TRIzol ${ }^{\circledR}$ were purchased from Invitrogen; Thermo Fisher Scientific, Inc. Anti- $\alpha$-tubulin and anti-tyrosinated tubulin antibodies were supplied by Sigma-Aldrich; Merck KGaA. The homologous recombination kit (cat. no. C122-02; Vazyme Biotech Co., Ltd.), DNA Polymerase (cat. no. P508-d1; Vazyme Biotech Co., Ltd.) and PrimeScript ${ }^{\mathrm{TM}}$ IV 1st strand cDNA synthesis mix (cat. no. 6215A; Takara Biotechnology Co., Ltd.) were used for construction of the recombinant plasmid, and the water used in the present study was ultra-pure $(R \geq 18 \mathrm{M}$ $\Omega / \mathrm{cm})$.

Cell culture and transfection. 293T and HeLa cells were purchased from Shanghai Institutes for Biological Sciences. The cells were routinely resuscitated, seeded into T25 cell culture flasks and cultured in DMEM high-glucose medium (containing $10 \% \mathrm{FBS}$ and $100 \mathrm{U} / \mathrm{ml}$ penicillin and streptomycin) at $37^{\circ} \mathrm{C}$ in a $5 \% \mathrm{CO}_{2}$ humidified atmosphere. At 70-80\% confluency, 293T and HeLa cells were transfected with $5 \mu \mathrm{g}$ GFP, Fign-GFP wild-type and mutant plasmids using a Lipofectamine ${ }^{\circledR} 2000$ transfection kit. Following a 30 -h incubation period at $37^{\circ} \mathrm{C}$ in a $5 \% \mathrm{CO}_{2}$ humidified atmosphere, the cells were harvested for western blotting.

Plasmid construction and mutagenesis. A construct containing the full-length coding sequence of Fign was synthesized from rat hippocampi using general PCR with gene-specific primers (listed in Table I) designed from the rat Fign gene (NM_001106484.1). PCR was performed as follows: $95^{\circ} \mathrm{C}$ for $3 \mathrm{~min}$, followed by 34 cycles of $95^{\circ} \mathrm{C}$ for $30 \mathrm{sec}, 59^{\circ} \mathrm{C}$ for $30 \mathrm{sec}$ and $72^{\circ} \mathrm{C}$ for $1 \mathrm{~min}$, then $72^{\circ} \mathrm{C}$ for $7 \mathrm{~min}$. The hippocampus was dissociated from four 1-day-old Sprague-Dawley rats. The rats were purchased from the animal experimental center of Sun Yat-sen University and immediately sacrificed upon arrival. Briefly, the newborn rats were exposed to carbon dioxide at $20 \%$ chamber replacement rate, and declared dead when the heart stopped beating, following which brain tissue was dissected. All animal procedures were performed in strict accordance with the recommendations in the Guide for the Care and Use of Laboratory Animals produced by the National Institutes of Health. The protocol was approved by the Institutional Animal Care and Use Committee at Jinan University. The gene was cloned into the BglII and EcoRI sites of pEGFP-N1 to generate the Fign-GFP plasmid. To construct the Fign-mNLS-GFP and Fign-DNLS-GFP plasmids, two-step PCR method was used to substitute or delete the basic amino acid residues in Fign. During the first PCR reaction step, complementary oligonucleotide primers were used to generate two DNA fragments with overlapping ends that contained the mutated or deleted bases. The overlapping ends were annealed, and the resulting fusion product served as a template for the second PCR reaction. Ligation of the mutant and deleted Fign fragments into the PEGFP-N1 vector was conducted using the homologous recombination kit, according to the manufacturer's protocols; colony PCR and agarose gel electrophoresis (1\% w/v agarose gel) experiments verified the fragments were successfully ligated to PEGFP-N1. Automated sequencing was used to confirm successful mutagenesis or deletion, and plasmid DNA was purified using the Plasmid Maxi kit (cat. no. 12163; Qiagen, Inc.), according to the manufacturer's instructions.

Western blotting. After transfection, the 293T and HeLa cells were lysed in lysis buffer [150 mM NaCl, $20 \mathrm{mM}$ Tris, $1 \mathrm{mM}$ EDTA, 1\% Triton X-100 and protease inhibitors cocktail (Sigma-Aldrich; Merck KGaA)] on ice for $30 \mathrm{~min}$. The lysates were centrifuged at $13,000 \mathrm{x}$ g for $15 \mathrm{~min}$ at $4^{\circ} \mathrm{C}$. A bicinchoninic acid protein assay was used to quantify the total protein concentration of each sample. The protein samples $(\sim 30 \mu \mathrm{g}$ /lane) were separated by SDS-PAGE on a $10 \%$ gel, and transferred to PVDF membranes (EMD Millipore). The membranes were blocked with $5 \%$ non-fat milk in TBS, $0.1 \%$ Tween-20 (TBST) at room temperature for $1 \mathrm{~h}$, and incubated overnight at $4^{\circ} \mathrm{C}$ with the following primary antibodies: Anti- $\alpha$-tubulin (1:2,000; cat.no. ab18251; rabbit polyclonal; Abcam); anti-tyrosinated tubulin $(1: 2,000$; cat. no. ab6160; rat monoclonal; Abcam); anti-GFP (1:5,000; cat. no. ab290; rabbit polyclonal; Abcam); anti-GAPDH (1:5,000; cat. no. ab181602; rabbit monoclonal; Abcam) and anti-Fign (1:400; cat. no. sc-68343; rabbit polyclonal; Santa Cruz Biotechnology, Inc.). After three washes in TBST, the membranes were incubated with horseradish peroxidase-conjugated donkey anti-rabbit (1:5,000; cat. no. AS038; ABclonal Biotech Co., Ltd.) or goat anti-mouse secondary antibodies (1:5,000; cat. no. AS003; ABclonal Biotech Co., Ltd.) at room temperature for $1 \mathrm{~h}$, and visualized using enhanced chemiluminescence reagents. Western blot bands were quantified by densitometric analysis using Image-Pro Plus 7.0 (Media Cybernetics, Inc.)

Immunocytochemistry assays. The HeLa cells were seeded onto glass cover slips (Thermo Fisher Scientific, Inc.) in 24-well 
Table I. Primer sequences for PCR.

\begin{tabular}{ll} 
Gene & \multicolumn{1}{c}{ Primer sequence $\left(5^{\prime} \rightarrow 3^{\prime}\right)$} \\
\hline Fign-GFP & F: GATTCTAGAGCTAGCGAATTCGCCACCATGCCGTACATCTTTGCCTTTT \\
Fign-GFP & R: GATCGCAGATCCTTCGCGGCCGCTTACAATCCTGTGGCTCCCAA \\
Fign-mNLS-GFP & F: CGGCAAGTTCTCTCAATAATAAAGCTTTCTATATGG \\
Fign-mNLS-GFP & R: CCATATAGAAAGCTTTATTATTGAGAGAACTTGCCG \\
Fign- $\Delta$ NLS-GFP & F: ACCAACAGTTCGGCAGCAGGGCAAGGAGAC \\
Fign- $\Delta$ NLS-GFP & R: GTCTCCTTGCCCTGCTGCCGAACTGTTGGT
\end{tabular}

Letters in underlined italics indicate induced restriction enzyme-recognized sites. F, forward; R, reverse; Fign, fidgetin; NLS, nuclear localization signal.

cell culture plates (Corning Inc.) at a density of $1 \times 10^{5} / \mathrm{ml}, 24 \mathrm{~h}$ prior to transfection. At a confluence of $40-50 \%$, HeLa cells were transfected with $1 \mu \mathrm{g} /$ well GFP, Fign-GFP wild-type and mutant plasmids using a Lipofectamine 2000 transfection kit. The transfection rate of HeLa cells in each group was $\sim 70 \%$. At $24 \mathrm{~h}$ post-transfection, the cells were fixed in $4 \%$ paraformaldehyde for $40 \mathrm{~min}$ at $4^{\circ} \mathrm{C}$, blocked with blocking buffer (TBST in 3\% normal donkey serum) at room temperature for $1 \mathrm{~h}$, and incubated overnight with an anti- $\alpha$-tubulin (1:200) or anti-tyrosinated tubulin (1:500) primary antibody at $4^{\circ} \mathrm{C}$. Next, the cells were incubated with a goat anti-mouse FITC secondary antibody (1:1,000; cat. no. ab150115; Abcam) for $2 \mathrm{~h}$ at room temperature. After staining, the cells were mounted on glass slides using Fluoro Gel II with DAPI (cat. no. 17985-50; Electron Microscopy Sciences) for confocal microscopy (LSM 700; Zeiss GmbH). Images were acquired with the same optical slice thickness in every channel using magnification x63 oil objective and a resolution of 1,024x1,024 pixels.

NLS prediction. Online prediction software (http://nls-mapper. iab.keio.ac.jp/cgi-bin/NLS_Mapper_form.cgi) was used to predict the Fign NLS. cNLS Mapper software accurately predicts NLSs specific to the importin $\alpha \beta$ pathway by calculating NLS scores (level of NLS activity); higher scores of Fign indicates stronger NLS activity.

Statistical analysis. Comparisons between different experimental groups were analyzed using one-way ANOVA. All data were tested for normal distribution; if the data were normally distributed and had similar variances, then one-way ANOVA was performed to compare the means among all measured variables followed by Bonferroni's post hoc test, $\mathrm{P}<0.05$ was considered to indicate a statistically significant difference. All statistical analyses were performed using SPSS version 12.0 software (SPSS, Inc.). Data are expressed as the mean \pm SEM of 3-5 separate experiments for each assay.

\section{Results}

Fign is predominantly located in the nucleus. In order to determine the cellular distribution of Fign, a eukaryotic expression vector containing the Fign coding sequence was constructed, which was amplified from rat cDNA. Colony PCR was used to determine successful insertion of the Fign sequence into the pEGFP-N1 plasmid. Fig. 1A shows the result of agarose gel electrophoresis of the representative colony PCR products. To further clarify whether Fign-GFP proteins were expressed from the corresponding plasmids, 293T cells were transfected with the Fign-GFP plasmids; western blotting with a GFP antibody revealed a major dominant band of the expected size ( 105 kDa; Fig. 1B). Next, the GFP epitope-tag was detected to delineate the subcellular localization of Fign in HeLa cells. With the exception of a small degree of cytoplasmic localization, the fusion protein was predominantly detected in the nucleus (Fig. 1C). However, no signal was detectable in the nucleoli of the GFP-positive cells, indicating that the overexpression of Fign-GFP fusion protein was localized to the nucleoplasm. Collectively, the results indicated that Fign is a nuclear protein.

A signal peptide sequence controls the translocation of Fign from the cytoplasm into the nucleus. Numerous studies have indicated that Fign is primarily involved in microtubule severing $(19,20)$, and the present study indicated that it is localized to the nucleus. As the NLS is an amino acid sequence that tags a protein for import into the nucleus, in the present study, the NLS of Fign was mutated to alter its cellular distribution, and the subsequent effects on microtubule severing were assessed. Using online NLS prediction software, the potential NLS of Fign was indicated to comprise amino acids $314-323$, a sequence that is relatively well conserved in rats, mice and humans (Fig. 2A). To further confirm that this putative sequence was a nuclear targeting sequence, the integrity of the NLS was disrupted by substituting amino acids 317 and 318 (KR to NN; Fign-mNLS-GFP), or removing the NLS entirely (Fign- $\Delta$ NLS-GFP; Fig. $2 B$ ). The sequencing results demonstrated that the mutagenesis was successful, and the colony PCR results showed bands in lanes 4 and 8 that were consistent with the molecular weight of Fign (Fig. 2C). The 293T cells were then transfected with the mutant plasmids, and Fign expression was detected by western blotting. Both proteins were successfully expressed in 293T cells (Fig. 2D).

In order to evaluate the potential contribution of the NLS to nuclear localization, HeLa cells were transfected with Fign-mNLS-GFP and Fign- $\triangle$ NLS-GFP. It was also revealed that the Fign-mNLS-GFP and Fign- $\Delta$ NLS-GFP mutants were expressed in both the cytoplasm and the nucleus, compared 

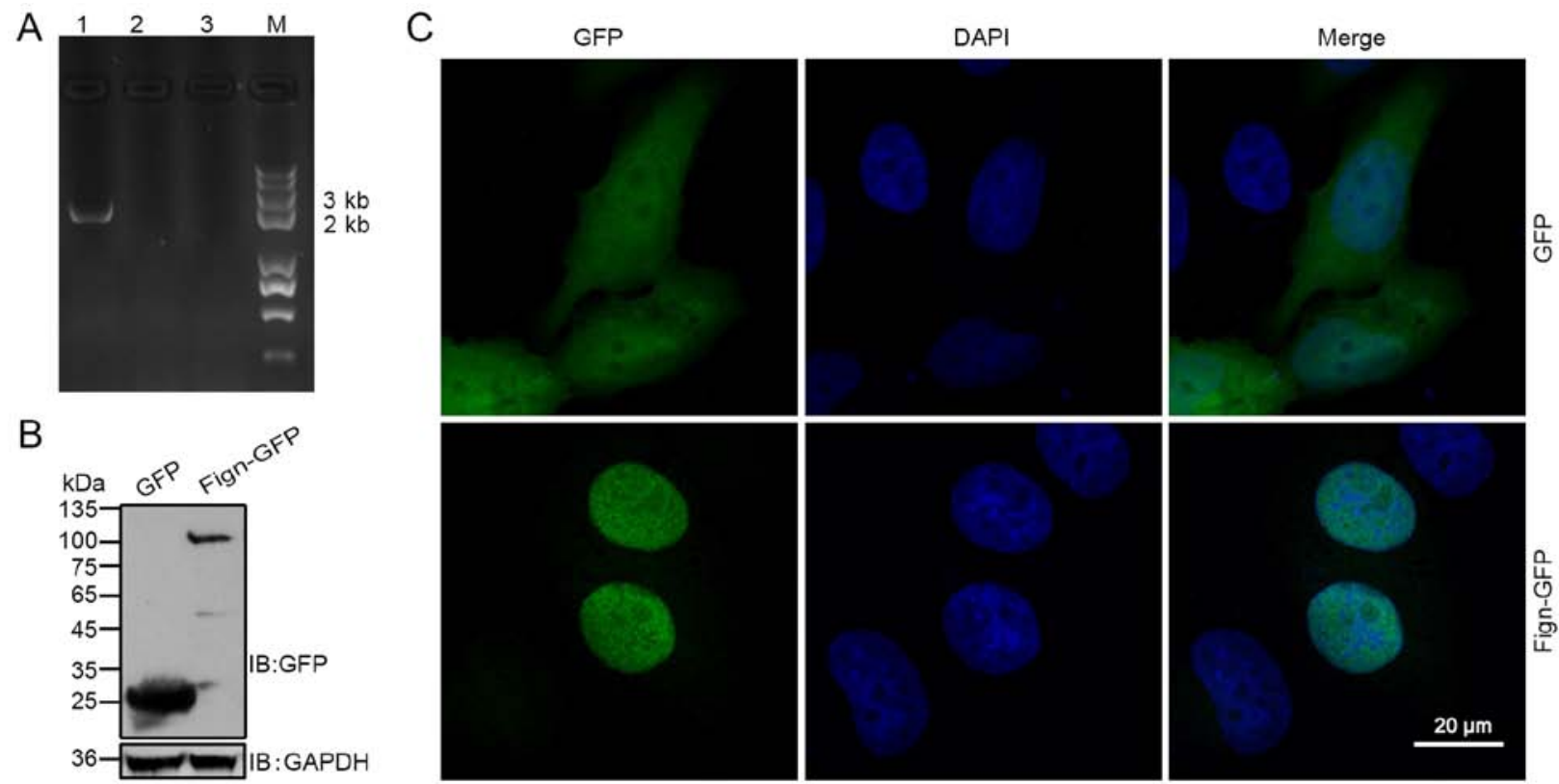

Figure 1. Construction of the Fign-GFP plasmid and subcellular localization of Fign primarily expressed in the nucleus. (A) Agarose gel electrophoresis of PEGFP-N1 universal primers. Lane 1, plasmid-positive colony of Fign-GFP; lanes 2-3, plasmid-negative colony; lane M, 2 kb - DNA ladder marker. (B) Fign protein expression detected by western blotting. (C) Subcellular localization of Fign-GFP in 293T cells. Fign, fidgetin.

A

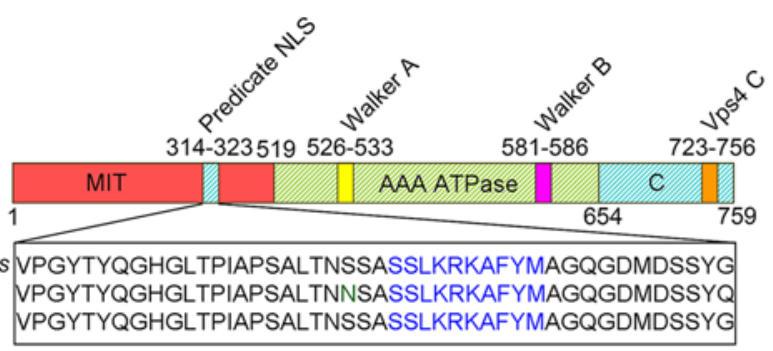

B

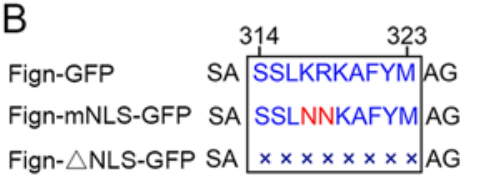

D

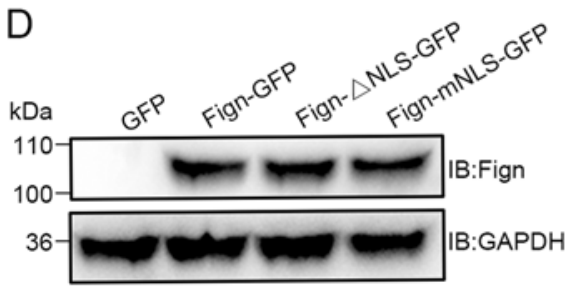

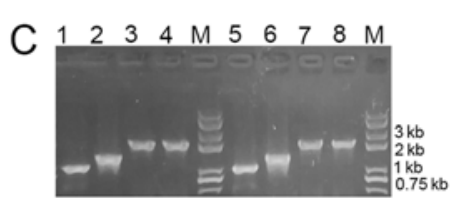

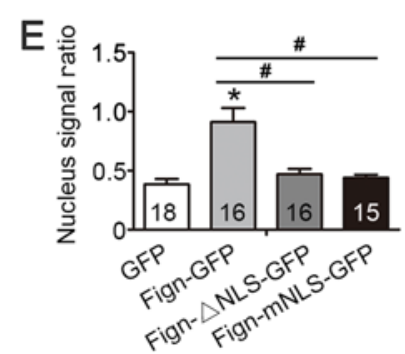

$\mathrm{F}$

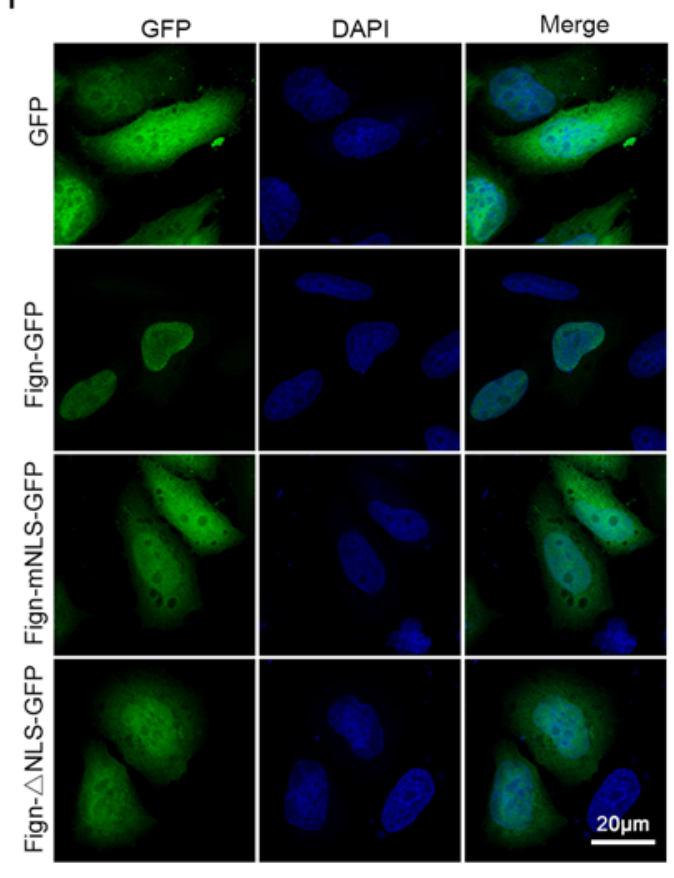

Figure 2. Schematic representation of the putative NLS and subcellular localization of Fign-mNLS-GFP and Fign- $\Delta$ NLS-GFP proteins. (A) Schematic representation of Fign protein and the putative NLS (amino acids 314-323). (B) Schematic representation of the NLS and its mutants. Fign-mNLS-GFP, KR (317-318) was replaced by NN; Fign- $\Delta$ NLS-GFP, the NLS was deleted. (C) Agarose gel electrophoresis of Fign and its fragments. Lane 4, Fign-mNLS-GFP colony PCR; lane 8, Fign- $\triangle$ NLS-GFP colony PCR; lanes 1 and 2, N-terminal fragments and C-terminal of Fign-mNLS-GFP; lanes 5 and 6, N-terminal and C-terminal fragments of Fign- $\Delta$ NLS-GFP; lanes 3 and 7, mNLS and $\triangle \mathrm{NLS}$ fragments of Fign. (D) Expression of mutant proteins was confirmed by western blotting. (E) Ratio of the average GFP signals in the nucleus and whole-cell. The cell and nuclear morphology were delineated under white light and DAPI conditions, respectively, and the gray value and area of the nuclei and intact cells were calculated by Image-Pro Plus 7.0 software. Nucleus signal ratio=nuclear grayscale mean/cell grayscale mean. (F) Fign and its mutant proteins expressed in HeLa cells. " $\mathrm{P}<0.05$ vs. Control group. " $\mathrm{P}<0.05$ vs. Control group. Fign, fidgetin; NLS, nuclear localization signal.

with cells transfected with wild-type Fign-GFP, whose protein was almost exclusively located in the nucleus (Fig. 2E and F). Taken together, the results indicated that the Fign NLS comprises amino acids 314-323 (N-terminal sequence, SSLKRKAFYM), and that K317 and R318 are key sites for the nuclear localization process. Furthermore, the mutation 
A
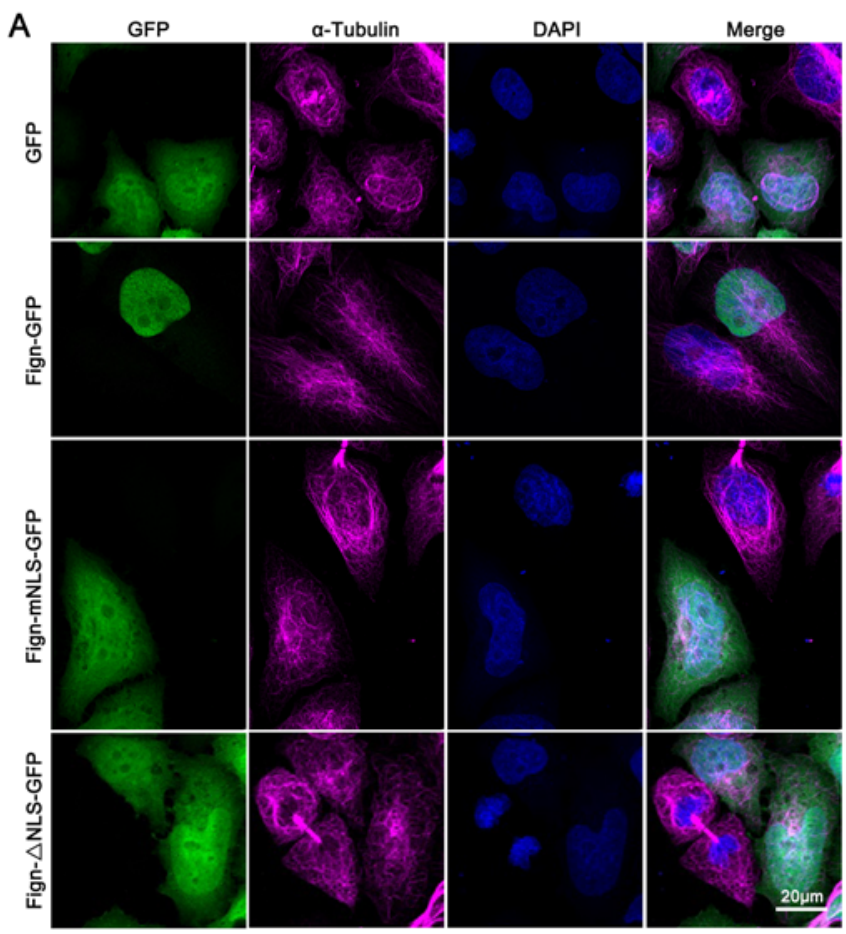

$\mathrm{B} \underset{\mathrm{s}}{\mathrm{s}}$

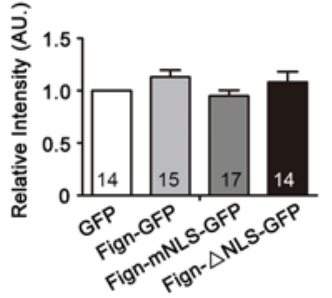

C

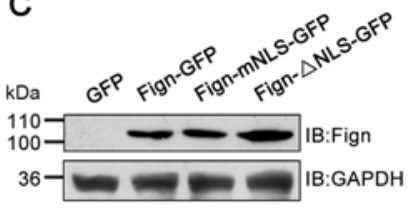

D
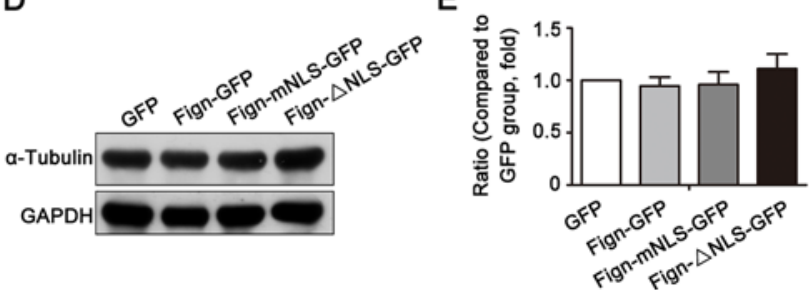

Figure 3. Fign and its mutants do not sever total microtubules. (A) Total microtubules in cells transfected with Fign and its mutants. (B) Quantification of the relative fluorescence intensity of $\alpha$-tubulin protein using Image-Pro Plus 7.0 software; compared to the GFP group, $\mathrm{n}=12$. (C) The expression of mutant protein was confirmed by western blotting. (D) Representative western blotting result showing that there are no change of $\alpha$-tubulin between Fign and its mutant. (E) Statistical data showing no change of $\alpha$-tubulin protein after Fign overexpression. The data are shown as mean \pm SE. Fign, fidgetin; NLS, nuclear localization signal.

or deletion of this potential NLS subsequently affected the translocation of Fign into the nucleus.

Fign preferentially severs tyr-MTs. To investigate whether Fign regulates microtubule severing, thus altering their dynamics, HeLa cells were selected to observe microtubule morphology. As shown in Fig. 3A, both Fign-GFP wild type and mutant plasmids were successfully expressed in HeLa cells. Because Fign is primarily expressed in the nucleus, the microtubules of the transfected cells showed no obvious morphological changes, and were not cut into short fragments. Also, there were no significant differences in fluorescence intensity or protein expression (Fig. 3A and D). Of note, no significant changes in $\alpha$-tubulin were observed in cells transfected with Fign-mNLS-GFP or Fign- $\triangle$ NLS-GFP compared with control cells (Fig. 3B and E). As Fign remained in the cytoplasm following NLS mutation or deletion, these data suggested that Fign did not alter microtubule dynamics by cutting the microtubules into short fragments.

Microtubules undergo various forms of post-translational modification. Since tyrosine modification increases microtubule instability, the microtubule-severing function of Fign in unstable microtubules was assessed. Immunocytochemistry assays and western blotting were used to detect tyr-MTs in the transfected cells. Although Fign was not responsible for cutting tyr-MTs into visibly shorter fragments, the fluorescence intensity and protein expression levels of these tyr-MTs were significantly reduced (Fig. 4A and C) compared with those of the control cells. Moreover, when cells were transfected with Fign-mNLS-GFP or Fign- $\triangle$ NLS-GFP, these parameters were also significantly reduced (Fig. 4B and D), collectively suggesting that Fign primarily exerts its effects on tyr-MTs.

\section{Discussion}

In the present study, online software was used to predict the potential NLS of Fign, and to identify its contribution to nuclear localization and microtubule severing. Since epitope-tagged Fign was predominantly localized to the nucleus, it was revealed to be a nuclear protein. Furthermore, amino acids K317 and R318 of the N-terminal sequence of Fign were identified to be necessary for its nuclear localization, and mutation of the NLS affected its nuclear transport. Moreover, Fign was indicated to preferentially sever tyr-MTs.

AAA proteins contain a highly conserved 230 amino-acid AAA domain, which forms hexameric rings essential for their ATPase activity (20-22). Similar to spastin and katanin, Fign efficiently severs microtubules in an ATP-dependent manner, resulting in their destruction $(9,23)$. By severing the lattice or removing tubulins directly from the tubule ends, microtubule-severing enzymes fulfill a key role in creating and maintaining microtubule arrays in a variety of cell types; creating new 'bare ends' renders microtubules highly responsive to other regulators in the local microenvironment (20).

The results of the present study provide important and complementary insights into the understanding of the nuclear export of Fign. Firstly, the N-terminal SSLKRKAFYM sequence (amino acids 314-323) was identified to be an NLS, enabling Fign to return to the cytoplasm from the nucleus. It 
A
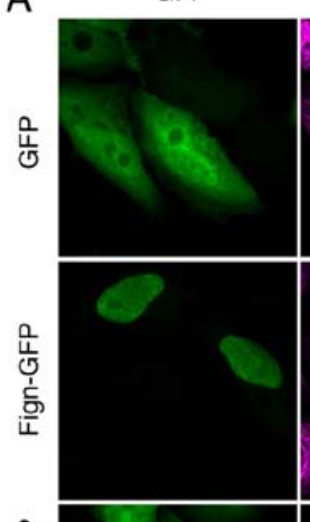

荘

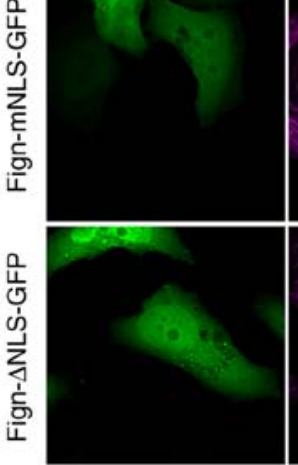

Tyr-tubulin
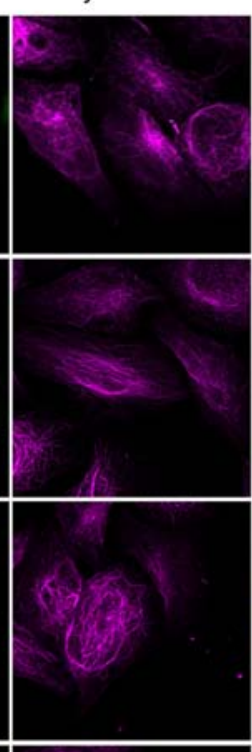
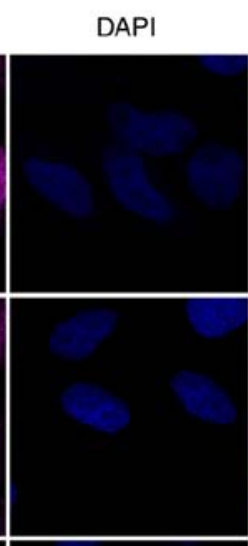
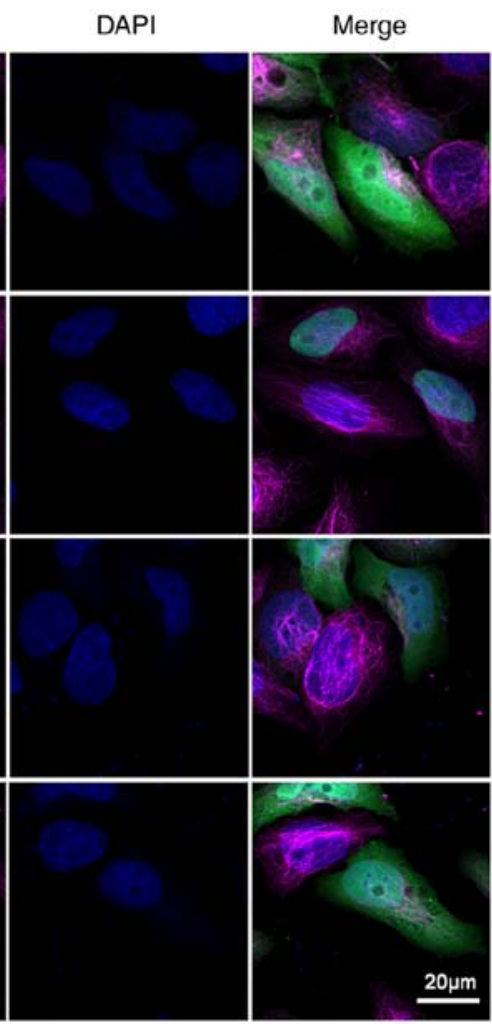

B

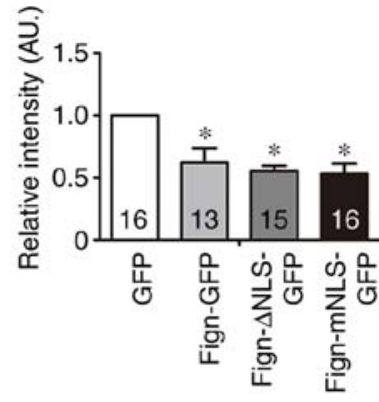

C
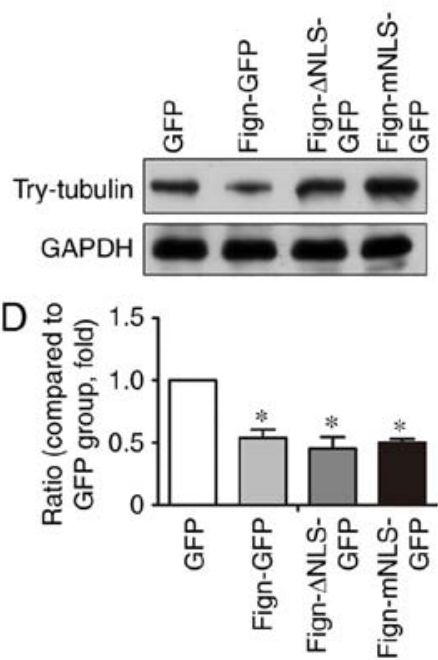

Figure 4. Fign and its mutants sever tyr-tubulin. (A) Tyr-tubulin in cells transfected with Fign and its mutants. (B) Quantification of the relative fluorescence intensity of tyr-tubulin protein using Image-Pro Plus 7.0 software; compared to the GFP group, n=12. (C) Representative western blotting result showing that Fign and its mutant overexpression cultures have a lower ratio of tyr-tubulin to $\alpha$-tubulin than GFP cultures. (D) Statistical data showing a decrease of tyr-tubulin protein after Fign and its mutants were overexpressed. The data are shown as the mean \pm SE. ${ }^{*} \mathrm{P}<0.05$ vs. Control group. Fign, fidgetin; tyr-, tyrosine; NLS, nuclear localization signal.

was also revealed that Fign is predominantly localized in the nucleus, and is thus a nuclear protein.

Nuclear localization is essential for controlling the transcription of protein-coding genes $(24,25)$. The majority of NLSs contain basic amino acid sequences of 4-30 residues, which are too short to form independent structures, and typically exist within nuclear proteins. The NLS is required for the transport of large proteins (>60 kDa) from or into the nucleus (26-28). As the NLS loses its capacity to bind to components within the nucleus, by default, the associated protein will be exported back into the cytoplasm (29-32). Since the molecular mass of Fign is $\sim 80 \mathrm{kDa}$, the Fign-GFP fusion protein exceeds the size of the nuclear pore complex (40-60 kDa; utilized for simple diffusion), suggesting that an active transport mechanism was necessary for the nuclear distribution of the fusion protein.

In order to identify the basic amino acid residues responsible for the nuclear localization of Fign, online software was used to predict its potential NLS. To generate Fign-NLS mutants, two conserved residues were then replaced, or the putative NLS was deleted entirely. Disruption of the NLS resulted in Fign expression in both the cytoplasm and the nucleus, indicating that the identified NLS was important for its cellular distribution. To identify the amino acid residues responsible for nuclear transport, a KR-to-NN mutation was generated in the Fign NLS, and its cellular distribution was assessed. The results showed that this mutation ablated nuclear translocation, and suggested that K317 and R318 are key residues for transportation regulation. Yang et al (11) have reported that Fign is a nuclear protein with a binary NLS that reveals two small clusters of essential amino acids (K366, K369, R378 and K379), similar to the canonical binary NLS. After comparison, the present study reported a different NLS than Yang et al (11).

A number of studies have emphasized the importance of the microtubule-severing function of Fign, which primarily influences microtubule dynamics $(19,20)$. It was also identified that Fign preferentially severs tyr-MTs (33). In addition to maintaining cell shape and structure, microtubules are involved in intracellular transport, cellular motility and the positioning of organelles (34). Microtubules are heterogeneous and highly dynamic both in vivo and in vitro, and undergo cycles of polymerization and rapid depolymerization (35). This 'dynamic instability' is an intrinsic property that has a key role in microtubule functioning (36). The present study revealed that, unlike spastin, another microtubule-severing protein, Fign, does not cut microtubules into short fragments, suggesting that it may not act on stable microtubules.

Although Fign does not have a significant effect on the shortening of intact, full-length microtubules, its influence on post-translationally modified microtubules remained to be elucidated. In order to determine whether Fign acted on modified microtubules, intracellular detection of tyr-MTs was conducted in the present study. The results showed that Fign and its mutants caused a significant decrease in the expression 
of tyr-MTs compared with wild-type Fign. It has been reported that tubulin is post-translationally modified in various ways, including phosphorylation, ubiquitination, sumoylation, and palmitoylation $(37,38)$. However, detyrosination, $\Delta 2$-tubulin generation, polyglutamylation, polyglycylation and acetylation occur less frequently. Different microtubule post-translational modifications, alone or in combination, alter the binding state of tubulin proteins and confer specific cellular functions (39-42). Detyrosination has been linked to microtubule stability, as stable microtubules are detyrosinated in multiple different cell types (43). Because tyrosine modification increases microtubule instability, the results of the current study suggested that Fign may preferentially sever tyrosine-modified and unstable, rather than stable, microtubules. Hu et al (33) reported that Fign has an essential role in cultured astrocyte migration by preferentially targeting tyr-MTs. The present study yielded consistent results with those of $\mathrm{Hu}$ et al (33). Before the experiment, it was hypothesized that mutations in the Fign NLS might lead to two outcomes. The first is that the mutation disrupts the function of Fign targeting the cleavage of tyr-MTs, resulting in an increase in tyr-MTs. The second is that the mutation does not affect the function of Fign, and the increased level of Fign in the cytoplasm enhances the cleavage of tyr-MTs. However, in fact, the Fign mutation did not result in a significant change in tyr-MTs compared with wild-type Fign, suggesting that the cellular distribution of the Fign protein itself does not affect the function of microtubule severing. Spastin and katanin, two other microtubule-severing proteins, destabilize local microtubule lattice contacts by pulling on the disordered and negatively-charged C-terminal tails of tubulin, which is dependent on the orientation of tubulin (44). As a microtubule-severing protein, Fign may utilize similar mechanisms.

In conclusion, the present study revealed that Fign is a nuclear protein that targeted and severed tyr-MTs, and that K317 and R318 (located at the N-terminus of the Fign gene) are key residues for its nuclear translocation. Further studies are required, however, to elucidate the mechanisms of the K317/R318-associated nuclear entry of Fign and the precise function of its signal peptide sequence.

\section{Acknowledgements}

Not applicable.

\section{Funding}

This work was supported by National Natural Science Foundation of China (grant nos. 81571191 and 81771144), Natural Science Foundation of Guangdong Province, China (grant nos. 2017B030311002 and 2017A030310342), Medical Research Foundation of Guangdong Province, China (grant no. A2016343) and Guangzhou Institute of Pediatrics/Guangzhou Women and Children's Medical Center (grant no. IP-2018-010).

\section{Availability of data and materials}

The datasets used and/or analyzed during the current study are available from the corresponding author on reasonable request.

\section{Authors' contributions}

JFZ, CC and GG conceived and designed the experiments. JL, FW and JQZ performed the experiments. JL and LFC analyzed the data. JL wrote the paper, LC and TF performed the western blotting. JFZ and GG critically revised the manuscript. All authors read and approved the final version of the manuscript.

\section{Ethics approval and consent to participate}

All animal procedures were performed in strict accordance with the recommendations in the Guide for the Care and Use of Laboratory Animals produced by the National Institutes of Health. The protocol was approved by the Institutional Animal Care and Use Committee at Jinan University.

\section{Patient consent for publication}

Not applicable.

\section{Competing interests}

The authors declare that they have no competing interests.

\section{References}

1. Fletcher DA and Mullins RD: Cell mechanics and the cytoskeleton. Nature 463: 485-492, 2010

2. Guerin CM and Kramer SG: Cytoskeletal remodeling during myotube assembly and guidance: Coordinating the actin and microtubule networks. Commun Integr Biol 2: 452-457, 2009.

3. Kapitein LC and Hoogenraad CC: Building the neuronal microtubule cytoskeleton. Neuron 87: 492-506, 2015.

4. Lasser M, Tiber J and Lowery LA: The role of the microtubule cytoskeleton in neurodevelopmental disorders. Front Cell Neurosci 12: 165, 2018.

5. Barlan K and Gelfand VI: Microtubule-based transport and the distribution, tethering, and organization of organelles. Cold Spring Harb Perspect Biol 9: pii: a025817, 2017.

6. Lipka J, Kuijpers M, Jaworski J and Hoogenraad CC: Mutations in cytoplasmic dynein and its regulators cause malformations of cortical development and neurodegenerative diseases. Biochem Soc Trans 41: 1605-1612, 2013.

7. Reiner $\mathrm{O}$ and Sapir T: LIS1 functions in normal development and disease. Curr Opin Neurobio 23: 951-956, 2013.

8. Liu Y, Lee JW and Ackerman SL: Mutations in the microtubule-associated protein 1A (Map1a) gene cause Purkinje cell degeneration. J Neurosci 35: 4587-4598, 2015.

9. Mukherjee S, Diaz Valencia JD, Stewman S, Metz J, Monnier S, Rath U, Asenjo AB, Charafeddine RA, Sosa HJ, Ross JL, et al: Human Fidgetin is a microtubule severing the enzyme and minus-end depolymerase that regulates mitosis. Cell Cycle 11: 2359-2366, 2012.

10. Ghosh DK, Dasgupta D and Guha A: Models, regulations, and functions of microtubule severing by Katanin. ISRN Mol Biol 2012: 596289, 2012.

11. Yang Y, Mahaffey CL, Bèrubè N, Nystuen A and Frankel WN: Functional characterization of fidgetin, an AAA-family protein mutated in fidget mice. Exp Cell Res 304: 50-58, 2005.

12. Cox GA, Mahaffey CL, Nystuen A, Letts VA and Frankel WN: The mouse fidgetin gene defines a new role for AAA family proteins in mammalian development. Nat Genet 26: 198-202, 2000.

13. Yang Y, Mahaffey CL, Bérubé N and Frankel WN: Interaction between fidgetin and protein kinase A-anchoring protein AKAP95 is critical for palatogenesis in the mouse. J Biol Chem 281: 22352-22359, 2006.

14. Eckert T, Le DT, Link S, Friedmann L and Woehlke G: Spastin's microtubule-binding properties and comparison to katanin. PLoS One 7: e50161, 2012. 
15. Grimm I, Saffian D, Platta HW and Erdmann R: The AAA-type ATPases Pex1p and Pex6p and their role in peroxisomal matrix protein import in Saccharomyces cerevisiae. Biochim Biophys Acta 1823: 150-158, 2012.

16. White SR and Lauring B: AAA+ ATPases: Achieving diversity of function with conserved machinery. Traffic 8: 1657-16672007.

17. Snider J, Thibault G and Houry WA: The AAA+ superfamily of functionally diverse proteins. Genome Biol 9: 216, 2008.

18. Onitake A, Yamanaka K, Esaki M and Ogura T: Caenorhabditis elegans fidgetin homolog FIGL-1, a nuclear-localized AAA ATPase, binds to SUMO. J Struct Biol 179: 143-151, 2012.

19. Leo L, Yu W, D'Rozario M, Waddell EA, Marenda DR, Baird MA, Davidson MW, Zhou B, Wu B, Baker L, et al: Vertebrate Fidgetin restrains axonal growth by severing labile domains of microtubules. Cell Rep 12: 1723-1730, 2015.

20. Sharp DJ and Ross JL: Microtubule-severing enzymes at the cutting edge. J Cell Sci 125: 2561-2569, 2012.

21. Lupas AN and Martin J: AAA proteins. Curr Opin Struct Biol 12: 746-753, 2002.

22. Vale RD: AAA proteins. J Cell Biol 150: F13-F19, 2000.

23. Zhang D, Rogers GC, Buster DW and Sharp DJ: Three microtubule severing enzymes contribute to the 'Pacman-flux' machinery that moves chromosomes. J Cell Biol 177: 231-242, 2007.

24. Spit A, Hyland RH, Mellor EJ and Casselton LA: A role for heterodimerization in nuclear localization of a homeodomain protein. Proc Natl Acad Sci USA 95: 6228-6233, 1998.

25. Hunter CC, Siebert KS, Downes DJ, Wong KH, Kreutzberger SD, Fraser JA, Clarke DF, Hynes MJ, Davis MA and Todd RB: Multiple nuclear localization signals mediate nuclear localization of the GATA transcription factor AreA. Eukaryot Cell 13: 527-538, 2014

26. LaCasse EC and Lefebvre YA: Nuclear localization signals overlap DNA- or RNA-binding domains in nucleic acid-binding proteins. Nucleic Acids Res 23: 1647-1656, 1995

27. Kosugi S, Hasebe M, Matsumura N, Takashima $H$ Miyamoto-Sato E, Tomita M and Yanagawa H: Six classes of nuclear localization signals specific to different binding grooves of importin alpha. J Biol Chem 284: 478-485, 2009.

28. Melnikov S, Ben-Shem A, Yusupova G and Yusupov M: Insights into the origin of the nuclear localization signals in conserved ribosomal proteins. Nat Commun 6: 7382, 2015.

29. Nakielny S and Dreyfuss G: Import and export of the nuclear protein import receptor transportin by a mechanism independent of GTP hydrolysis. Curr Biol 8: 89-95, 1998.

30. Wang $R$ and Brattain MG: The maximal size of protein to diffuse through the nuclear pore is larger than $60 \mathrm{kDa}$. FEBS Lett 581: 3164-3170, 2007.
31. Freitas $\mathrm{N}$ and Cunha $\mathrm{C}$ : Mechanisms and signals for the nuclear import of proteins. Curr Genomics 10: 550-557, 2009.

32. Marfori M, Mynott A, Ellis JJ, Mehdi AM, Saunders NF, Curmi PM, Forwood JK, Bodén M and Kobe B: Molecular basis for specificity of nuclear import and prediction of nuclear localization. Biochim Biophys Acta 1813: 1562-1577, 2011.

33. Hu Z, Feng J, Bo W, Wu R, Dong Z, Liu Y, Qiang L and Liu M: Fidgetin regulates cultured astrocyte migration by severing tyrosinated microtubules at the leading edge. Mol Biol Cell 28: 545-553, 2017.

34. Kaverina I and Straube A: Regulation of cell migration by dynamic microtubules. Semin Cell Dev Biol 22: 968-974, 2011.

35. Muroyama A and Lechler T: Microtubule organization, dynamics and functions in differentiated cells. Development 144: 3012-3021, 2017.

36. Mitchison T and Kirschner M: Dynamic instability of microtubule growth. Nature 312: 237-242, 1984.

37. Song Y and Brady ST: Post-translational modifications of tubulin: Pathways to functional diversity of microtubules. Trends Cell Biol 25: 125-136, 2015.

38. Audagnotto $\mathrm{M}$ and Dal Peraro M: Protein post-translational modifications: In silico prediction tools and molecular modeling. Comput Struct Biotechnol J 15: 307-319, 2017.

39. Howes SC, Alushin GM, Shida T, Nachury MV and Nogales E: Effects of tubulin acetylation and tubulin acetyltransferase binding on microtubule structure. Mol Biol Cell 25: 257-266, 2014.

40. Magiera MM and Janke C: Post-translational modifications of tubulin. Curr Biol 24: R351-R354, 2014.

41. Janke C and Bulinski JC: Post-translational regulation of the microtubule cytoskeleton: Mechanisms and functions. Nat Rey Mol Cell Biol 12: 773-786, 2011.

42. Fukushima N, Furuta D, Hidaka Y, Moriyama R and Tsujiuchi T: Post-translational modifications of tubulin in the nervous system. J Neurochem 109: 683-693, 2009.

43. Schneider N, Ludwig H and Nick P: Suppression of tubulin detyrosination by parthenolide recruits the plant-specific kinesin KCH to cortical microtubules. J Exp Bot 66: 2001-2011, 2015.

44. Roll-Mecak A and Vale RD: Structural basis of microtubule severing by the hereditary spastic paraplegia protein spastin. Nature 451: 363-367, 2008.

This work is licensed under a Creative Commons Attribution-NonCommercial-NoDerivatives 4.0 International (CC BY-NC-ND 4.0) License. 